\title{
Central-to-peripheral systolic blood pressure different phenotypes and relation to accuracy of daily used cuff devices
}

\author{
Ahmed Bendary', Mina Magdy², Sahar Abdoul Aal', Hisham Rashid ${ }^{1}$ \\ ${ }^{1}$ Banha Faculty of Medicine, Banha University, Banha, Egypt \\ ${ }^{2}$ National Heart Institute, Giza, Egypt
}

\begin{abstract}
Background. Cuff blood pressure (BP) measurement has been the standard method for taking BP in routine daily practice for more than a century. However, some concerns were raised about the accuracy of this method which could lead to misclassification of BP in many situations.

We aimed primarily to confirm a recent major discovery that distinct BP phenotypes based on central-to-peripheral systolic blood pressure (SBP) amplification do exist, and whether application of a validated cuff BP method (e.g. oscillometric) could accurately discriminate these differences.

Material and methods. Among 106 participants (mean age $62 \pm 11 ; 58 \%$ males) undergoing coronary angiography, intra-arterial BP was measured at 3 points (ascending aorta, brachial and radial arteries). Central-to-peripheral SBP amplification (SBPamp) was defined as $\geq 5 \mathrm{~mm} \mathrm{Hg} \mathrm{SBP}$ increased from aorta-to-brachial and/or from brachial-to-radial arteries. A validated cuff BP device (oscillometric) was used to measure BP at 4 different time points.

Results. Four different BP phenotypes were confirmed based on the magnitude of SBPamp; phenotype I, both aortic-to brachial and brachial-to radial SBPamp; phenotype II, only aortic-to-brachial SBPamp; phenotype III, only brachial-to-radial SBPamp; and phenotype IV, no SBPamp at all. Aortic SBP was significantly higher in phenotypesIII and IV compared to phenotypes-I and II ( $\mathrm{p}=0.001)$. This was not discriminated using a validated cuff BP device measurement $(\mathrm{p}=0.996)$. Results for the pulse pressure (PP) followed the same pattern.

Conclusion. Distinct BP phenotypes do exist based on SBPamp. A validated cuff BP method failed to discriminate this. Improving quality of BP measurements in daily practice is a priority.

Key words: blood pressure; catheterization; phenotypes; cuff devices
\end{abstract}

Arterial Hypertens. 2019, vol. 23, no. 4, pages: 263-270

DOI: 10.5603/AH.a2019.0019

\section{Introduction}

Blood pressure (BP) measurement in a proper way is regarded as one of the most important measurements in daily clinical practice [1]. This is because accurate diagnosis of hypertension (HTN), accompanied by lifestyle modifications (with or without anti-hypertensive medications) can lead to substantial reductions of the future cardiovascular $(\mathrm{CV})$ risk [2]. Despite many concerns raised about the accuracy of cuff BP measurements used in daily practice [3], this method has not changed significantly over the past century. Thanks to a recent individual patient data systematic review and meta-analysis [4], it has

Address for correspondence: Dr. Ahmed Bendary, MD

Cardiology Department, Benha Faculty of Medicine, Benha University, Egypt, Postal Code no. 13518

e-mail: ahmed.bendari@fmed.bu.edu.eg;dr_a_bendary@hotmail.com 
been shown that routinely used cuff BP devices are exposed to inaccuracies when compared to intra-arterial (invasive) BP values, especially for systolic blood pressure (SBP) in the range of 120-159 $\mathrm{mm} \mathrm{Hg}$. Accordingly, this could have huge implications on how HTN is correctly diagnosed in the first place.

Cuff BP devices (both oscillometric and standard Korotkoff sounds) use arterial signals at an isolated point in a peripheral artery (usually the brachial artery) to measure BP $[5,6]$. However, there are large inter-individual variability in arterial characteristics (e.g. stiffness, diameter, etc.) and in the way BP is transmitted from central-to-peripheral vasculature (e.g. increased SBP amplification in some, but not in others) [7-10]. Therefore, measuring BP at an isolated arterial point may overlook these fine phenotypic differences.

This notion has been recently raised by Picone et al [11], who discovered for the first time that there are 4 distinctive phenotypes of BP (based on central-to-peripheral SBP amplification as measured invasively), and that these different phenotypes were not discriminated at all by cuff BP devices used in daily routine practice. Importantly, they cautioned that these findings may not be generalizable to other patients beyond their study population and that future research is warranted to corroborate these findings and to test whether such phenotypes would be present among sample populations other than those examined in their work. Therefore, we found that it may be of considerable interest if we could replicate these novel findings among a group of patients with different ethnicity and characteristics.

\section{Material and methods}

\section{Study subjects}

In the period from November 2018 to April 2019, this observational study included patients with a clinical indication for coronary angiography at National Heart Institute, Giza, Egypt. Among 181 patients screened, seventy-five patients were excluded leaving 106 eligible patients for study procedures. Patients were excluded with the following criteria that may introduce errors in intra-arterial BP measurement; atrial fibrillation or aortic stenosis $(\mathrm{n}=16)$; a cuff $\mathrm{BP}$ inter-arm difference of $>5 \mathrm{~mm} \mathrm{Hg}(\mathrm{n}=8)$; inability to measure BP in both arms for whatever reason $(\mathrm{n}=10)$; use of femoral arterial access for coronary angiography (due to inaccessibility of upper limb arteries; $\mathrm{n}=11$ ). We also excluded patients with medical (radial artery spasm; $n=6$ ) or technical issues that detracted the quality of pressure waveforms recorded $(\mathrm{n}=5)$. Nineteen patients did not provide a consent. Included patients' clinical characteristics including anthropometric measures and clinical history were recorded at the angiography preassessment (usually 1 day before the procedure) by nursing staff. Other clinical information was extracted from the hospital medical records. The study was approved by the local ethical committee and all participants provided an informed consent.

\section{Intra-arterial BP measurement}

The right radial artery was used for catheter access. All patients were examined under stable haemodynamics without moving or talking. They were also devoid of any medications that could induce an acute vasoactive response as per the ARTERY guidelines (Association for Research into Arterial Structure and Physiology) [12]. After the clinical procedure, intraarterial BP was measured with a fluid-filled system using $5 \mathrm{~F}$ (18\% of cases) and 6F (82\% of cases) catheters, including a 5-6F Judkins Left (Cordis) and 5-6F Multipurpose (Cordis) catheters. Fluoroscopy was used to confirm the catheter position at each arterial site and the catheter was flushed before all waveform recordings. Stable pressure waveforms were recorded for a minimum of 20 seconds at each arterial site to reduce the influence of respiratory variation. Intra-arterial BP measurements began with the catheter in the proximal ascending aorta. The catheter was then pulled back to the upper arm (midhumerus) for brachial waveform measurement. Finally, the catheter was pulled back to the wrist, and the sheath was partially removed to allow the most distal radial waveform measurement possible. Waveforms were recorded via Dräger, Infinity Delta series, Germany.

\section{Cuff BP measurement}

Because BP value may change over time, cuff BP was measured at 4 different time points (for each patient): first, during the angiography preassessment visit; second, in the waiting room before catheterization, simultaneously on both arms with 2 identical devices; third, immediately before commencing the angiogram in the catheterization laboratory; and fourth, after the clinical procedure simultaneous with intra-arterial brachial BP. For all cuff measurements, participants were asked to remain still and quiet throughout the recordings. Cuff BP was measured with the automated oscillometric method using Omron HEM-907 device (which has been validated according to international protocols [13]). For cuff and intra-arterial measurements, pulse pressure (PP) was 
calculated as $\mathrm{SBP}$ - diastolic BP. Mean arterial pressure (MAP) was calculated as $\mathrm{DBP}+1 / 3 \mathrm{PP}$.

\section{Defining BP phenotypes}

As stated in Picone et al [11] methodology, the amount of SBP increase (SBP amplification) between the aortato-brachial and brachial-to-radial arteries was used to define the BP phenotypes. When SBP increased by $\geq 5$ $\mathrm{mm} \mathrm{Hg}$ between the aortic- to-brachial or brachial-toradial arteries, this was considered an SBP amplification. The threshold of $5 \mathrm{~mm} \mathrm{Hg}$ was selected because it represents a difference in BP generally greater than what could be attributed to measurement variability $[14,15]$. SBP increases of $<5 \mathrm{~mm} \mathrm{Hg}$ between the aortic-to-brachial or brachial-to-radial arteries were not considered an SBP amplification. Phenotypic differences' delineation employs SBP specifically because SBP is more closely related to cardiovascular disease and outcomes compared with other BP indices [16, 17]. Moreover, there is little variability in diastolic BP between the central-to-peripheral arteries [4].

\section{Statistical analysis}

Data management and statistical analysis were done using SPSS vs.25. (IBM, Armonk, New York, United States). Numerical data was summarized as means and standard deviations or medians and ranges. Categori- cal data was summarized as numbers and percentages. Comparisons between four groups were done using One-way ANOVA or Kruskal-Wallis test for normally and non-normally distributed numerical variables respectively. Post hoc analysis was done in case of significant overall effect. All post hoc analysis was Bonferroni adjusted. Categorical data was compared using Chi-square test. All p values were two sided. $P$ values less than 0.05 were considered significant.

\section{Results}

\section{Study population}

Generally, participants' criteria were typically those of patients undergoing coronary catheterization: middle to older age (mean age $62 \pm 11$ years), with male gender preponderance $(61 \%)$ and overweight to obese [mean body mass index (BMI) $30 \pm 4 \mathrm{~kg} / \mathrm{m}^{2}$ ]. Between-group analysis showed no statistically significant differences in all baseline characteristics (except for age) (Tab. I).

\section{$\mathrm{BP}$ phenotypes and cuff $\mathrm{BP}$ values}

Four different BP phenotypes were observed according to the amount of aortic-to-brachial and brachialto-radial SBP amplification (Fig. 1):

Table I. Baseline characteristics of study population*

\begin{tabular}{|c|c|c|c|c|c|c|}
\hline & & $\begin{array}{c}\text { Phenotype I } \\
(\mathrm{n}=27)\end{array}$ & $\begin{array}{l}\text { Phenotype II } \\
(\mathbf{n}=29)\end{array}$ & $\begin{array}{l}\text { Phenotype III } \\
(\mathrm{n}=26)\end{array}$ & $\begin{array}{l}\text { Phenotype IV } \\
(\mathbf{n}=24)\end{array}$ & $\begin{array}{c}p \\
\text { value }\end{array}$ \\
\hline Age (years) & Mean \pm SD & $56 \pm 10^{a}$ & $58 \pm 8^{a}$ & $69 \pm 9^{b}$ & $68 \pm 9^{b}$ & $<0.001$ \\
\hline \multirow{2}{*}{ Gender } & $\begin{array}{l}\text { Males } \\
\mathrm{n}(\%)\end{array}$ & $15(55.6)$ & $19(65.5)$ & $14(53.8)$ & $13(54.2)$ & 0.788 \\
\hline & $\begin{array}{l}\text { Females } \\
\mathrm{n}(\%)\end{array}$ & $12(44.4)$ & $10(34.5)$ & 12 (46.2) & $11(45.8)$ & \\
\hline Hypertension & $\begin{array}{l}\text { Yes } \\
\mathrm{n}(\%)\end{array}$ & $18(66.7)$ & $18(62.1)$ & $16(61.5)$ & $16(66.7)$ & 0.965 \\
\hline Diabetes & $\begin{array}{l}\text { Yes } \\
\mathrm{n}(\%)\end{array}$ & $12(44.4)$ & 12 (41.4) & 12 (46.2) & $10(41.7)$ & 0.982 \\
\hline Smoking & $\begin{array}{l}\text { Yes } \\
\mathrm{n}(\%)\end{array}$ & $10(37.0)$ & $12(41.4)$ & $10(38.5)$ & $10(41.7)$ & 0.982 \\
\hline Obesity & $\begin{array}{l}\text { Yes } \\
\mathrm{n}(\%)\end{array}$ & $8(29.6)$ & $9(31.0)$ & $8(30.8)$ & $8(33.3)$ & 0.994 \\
\hline BMI $\left[\mathrm{kg} / \mathrm{m}^{2}\right]$ & Mean \pm SD & $30 \pm 4$ & $30 \pm 3$ & $30 \pm 3$ & $31 \pm 5$ & 0.845 \\
\hline Known dyslipidaemia & $\begin{array}{l}\text { Yes } \\
\mathrm{n}(\%)\end{array}$ & $5(18.5)$ & $6(20.7)$ & $5(19.2)$ & $5(20.8)$ & 0.996 \\
\hline FH of premature CAD & $\begin{array}{l}\text { Yes } \\
\mathrm{n}(\%)\end{array}$ & $6(22.2)$ & $7(24.1)$ & $6(23.1)$ & $6(25.0)$ & 0.996 \\
\hline PH of CVD & $\begin{array}{l}\text { Yes } \\
\mathrm{n}(\%)\end{array}$ & $20(74.1)$ & $23(79.3)$ & $20(76.9)$ & $18(75.0)$ & 0.97 \\
\hline
\end{tabular}

*One-way ANOVA was used for age and BMI. Chi-square test was used for categorical data. Post hoc analysis was done in case of significant overall effect and different letters indicate significant pair. All post hoc were Bonferroni adjusted; $\mathrm{BMI}$ - body mass index; $\mathrm{CAD}$ - coronary artery disease; $\mathrm{FH}$ - family history; $\mathrm{PH}$ - past history; CVD — cardiovascular disease; $\mathrm{SD}$ — standard deviation 




Figure 1. Flow-chart of identification of various blood pressure (BP) phenotypes

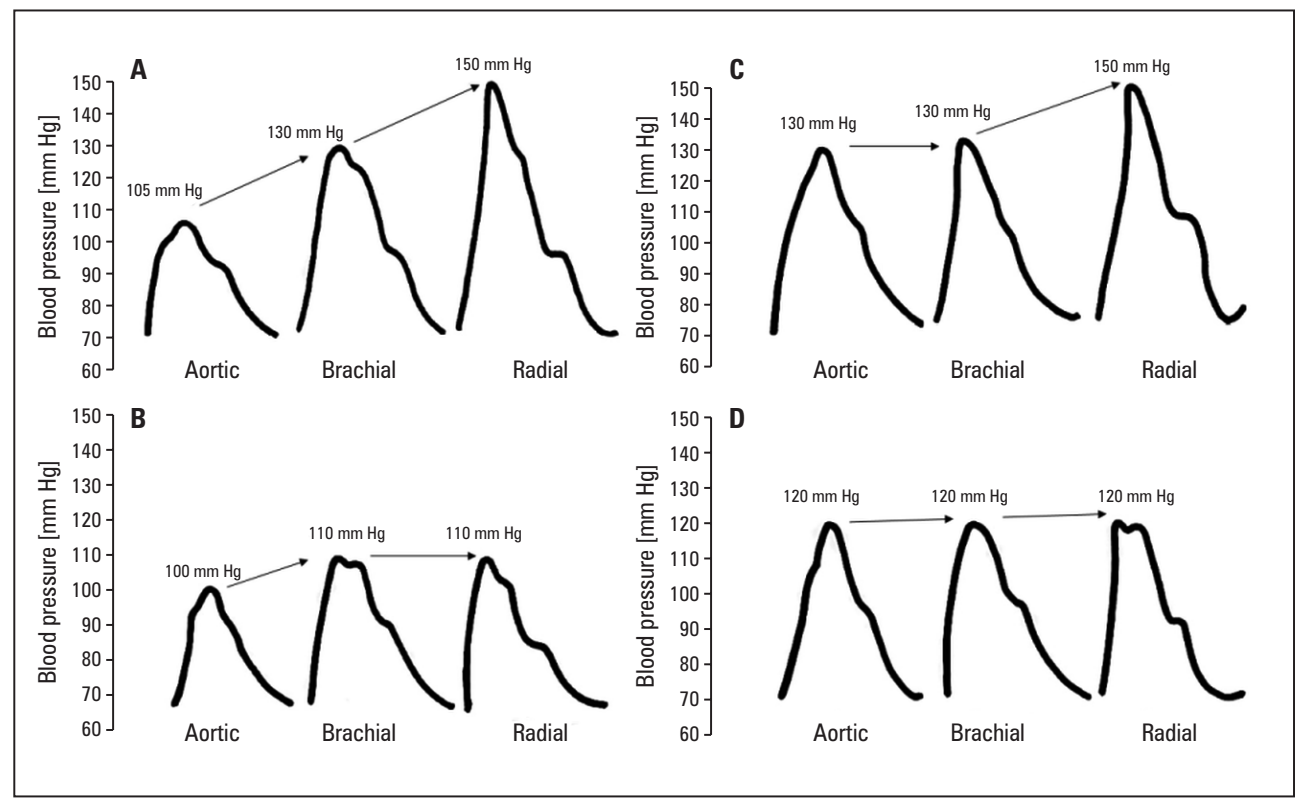

Figure 2. Case presentations of the 4 different blood pressure (BP) phenotypes based on differences in systolic blood pressure (SBP) amplification between aortic-to brachial and brachial-to-radial arteries. Recorded SBP is reported above each pressure waveform.

A. Phenotype I (case no. 11), B. Phenotype II (case no. 3); C. Phenotype III (case no. 17); D. Phenotype IV (case no. 14)

- both aortic-to-brachial and brachial-to-aortic SBP amplification $(\geq 5 \mathrm{~mm} \mathrm{Hg})$ in 27 patients (25.47\%; phenotype I) (Fig. 2A);

- aortic-to-brachial but not brachial-to-radial SBP amplification in 29 patients (27.35\%; phenotype II) (Fig. 2B);

- no aortic-to-brachial, but brachial-to-radial SBP amplification in 26 patients $(24.52 \%$; phenotype III) (Fig. 2C);
- neither aortic-to-brachial nor brachial-to-radial SBP amplification in 24 patients $(22.64 \%$; phenotype IV) (Fig. 2D).

Aortic SBP was significantly different between the $4 \mathrm{BP}$ phenotypes (overall $\mathrm{p}$ value $=0.001$ ) and was significantly higher in phenotypes-III and IV when compared to phenotype I (Fig. 3A; Tab. II). On the other hand, there was no statistically significant differences between groups as regard brachial SBP (Fig. 


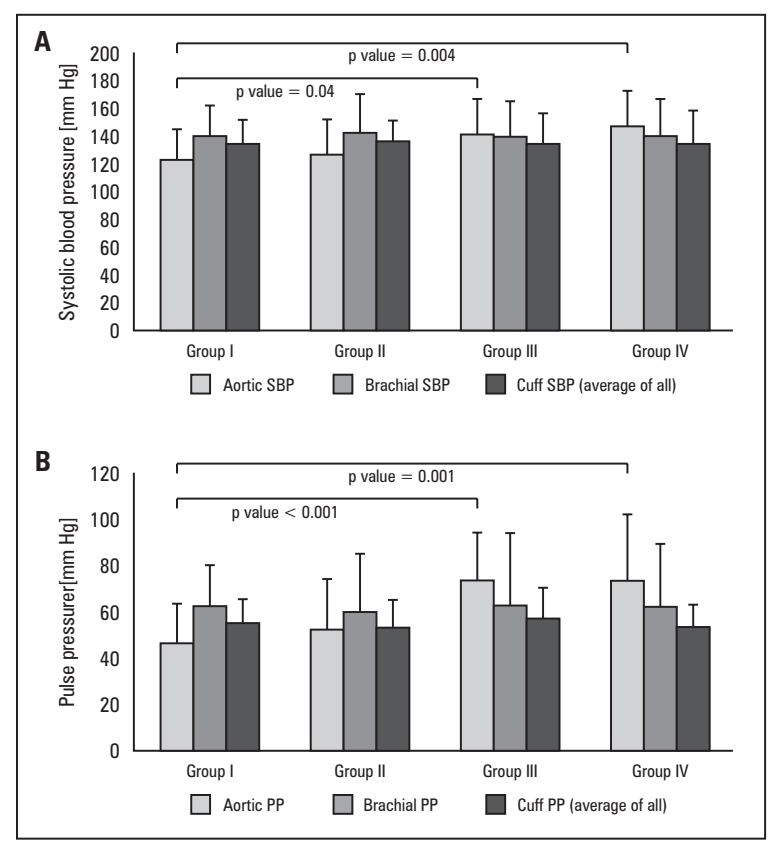

Figure 3. Cuff, intra-arterial aortic, and brachial blood pressure (BP) across the 4 BP phenotypes. A. Systolic BP (SBP); B. Pulse pressure (PP) - measured at the aorta (light bars), brachial artery (striated bars), and cuff BP measures (dark bars) across each BP phenotype. $p$ values on the figure represent significant differences in aortic SBP and PP between the specific phenotypes. $p>0.05$ for all other comparisons. Data are mean $\pm \mathrm{SE}$ of the mean

3A). Despite this marked variation in SBP amplification from central-to-peripheral vasculature across the 4 distinct BP phenotypes, there was no significant difference in standard cuff SBP measurements between the 4 groups (Tab. III). Of note, this lack of discrimination was consistent across all the 4 time points for cuff BP measurement and for the average of all cuff BP readings ( $\mathrm{p}$ values $>0.50$ for all comparisons; Fig. 3A; Tab. III).

Findings for PP followed the same pattern as for SBP; aortic PP was significantly different across the 4 BP phenotypes (Fig. 3B; Tab. II), but cuff BP measurement did not discriminate this accurately whether measures at different time points of using the average of all cuff BP readings ( $\mathrm{p}$ values $>0.30$ for all comparisons; Fig. 3B; Tab. III).

\section{Discussion}

To the best of our knowledge, this study is the $1^{\text {st }}$ in the literature that reaffirms the major findings provided by Picone et al [11] who discovered the presence of 4 different BP phenotypes based on the magnitude of central-to-peripheral SBP amplification. Notably, these various phenotypes were not discriminated by cuff BP measurements as used in routine daily practice. These findings make sense and imply that the practice of using standard one-for-all cuff BP devices should be revisited.

The clinical consequences of these findings are many, failure of a daily used cuff BP device to accurately discriminate the higher intra-arterial SBP (and PP) among phenotypes III and IV (groups with little or no aortic-to-brachial SBP amplification) could lead to misclassification of BP and potentially missing the opportunity of implementing lifestyle changes or even initiating antihypertensive medications.

Table II. Haemodynamic parameters across the 4 study groups*

\begin{tabular}{|l|l|c|c|c|c|c|}
\hline & \multicolumn{1}{|c|}{$\begin{array}{c}\text { Group I } \\
(\mathbf{n}=\mathbf{2 7})\end{array}$} & $\begin{array}{c}\text { Group II } \\
(\mathbf{n}=29)\end{array}$ & $\begin{array}{c}\text { Group III } \\
(\mathbf{n}=\mathbf{2 6})\end{array}$ & $\begin{array}{c}\text { Group IV } \\
\text { (n=24) }\end{array}$ & p value \\
\hline Aortic SBP [mm Hg] & Mean \pm SD & $122 \pm 22^{\mathrm{a}}$ & $126 \pm 26^{\mathrm{a}, \mathrm{c}}$ & $140 \pm 26^{\mathrm{b}, \mathrm{c}}$ & $146 \pm 24^{\mathrm{b}}$ & 0.001 \\
\hline Brachial SBP [mm Hg] & Mean \pm SD & $139 \pm 22$ & $141 \pm 28$ & $139 \pm 26$ & $140 \pm 26$ & 0.988 \\
\hline Radial SBP [mm Hg] & Mean \pm SD & $156 \pm 29^{\mathrm{a}}$ & $140 \pm 29^{\mathrm{b}, \mathrm{c}}$ & $156 \pm 39^{\mathrm{a}, \mathrm{c}}$ & $137 \pm 25^{\mathrm{b}, \mathrm{d}}$ & 0.038 \\
\hline $\begin{array}{l}\text { Aortic-to-brachial } \\
\text { SBPamp [mm Hg] }\end{array}$ & $\begin{array}{l}\text { Median } \\
\text { (range) }\end{array}$ & $15(5-55)^{\mathrm{a}}$ & $10(5-50)^{\mathrm{a}}$ & $0(-5-0)^{\mathrm{b}}$ & $0(-10-0)^{\mathrm{b}}$ & $<0.001$ \\
\hline $\begin{array}{l}\text { Brachial-to radial } \\
\text { SBPamp [mm Hg] }\end{array}$ & $\begin{array}{l}\text { Median } \\
\text { (range) }\end{array}$ & $10(5-60)^{\mathrm{a}}$ & $0(-10-0)^{\mathrm{b}}$ & $20(5-60)^{\mathrm{a}}$ & $0(-5-2)^{\mathrm{b}}$ & $<0.001$ \\
\hline Aortic MAP [mm Hg] & Mean \pm SD & $93 \pm 16$ & $93 \pm 15$ & $94 \pm 16$ & $92 \pm 13$ & 0.961 \\
\hline Aortic DBP [mm Hg] & Mean \pm SD & $76 \pm 16$ & $74 \pm 13$ & $72 \pm 13$ & $73 \pm 12$ & 0.739 \\
\hline Aortic PP [mm Hg] & Mean \pm SD & $46 \pm 17^{\mathrm{a}}$ & $52 \pm 21^{\mathrm{a}}$ & $73 \pm 21^{\mathrm{b}}$ & $73 \pm 28^{\mathrm{b}}$ & $<0.001$ \\
\hline Brachial PP [mm Hg] & Mean \pm SD & $62 \pm 17$ & $60 \pm 24$ & $62 \pm 31$ & $61 \pm 28$ & 0.997 \\
\hline Radial PP [mm Hg] & Mean \pm SD & $77 \pm 24$ & $65 \pm 26$ & $79 \pm 26$ & $63 \pm 30$ & 0.05 \\
\hline Heart rate [bpm] & Mean \pm SD & $80 \pm 11$ & $81 \pm 9$ & $80 \pm 11$ & $81 \pm 10$ & 0.978 \\
\hline
\end{tabular}

${ }^{*}$ One-way ANOVA was used. Kruskal Wallis test was used for aortic to brachial SBPamp and brachial to radial SBPamp. Post hoc analysis was done in case of significant overall effect and different letters indicate significant pair. All post hoc were Bonferroni adjusted; Bpm — beat per minute; DBP — diastolic blood pressure; MAP — mean arterial pressure; PP — pulse pressure; SBPamp — systolic blood pressure amplification 
Table III. Cuff blood pressure (BP) across the 4 study groups*

\begin{tabular}{|c|c|c|c|c|c|}
\hline & $\begin{array}{l}\text { Group I } \\
(\mathrm{n}=27)\end{array}$ & $\begin{array}{l}\text { Group II } \\
(n=29)\end{array}$ & $\begin{array}{l}\text { Group III } \\
(n=26)\end{array}$ & $\begin{array}{l}\text { Group IV } \\
(n=24)\end{array}$ & $\mathrm{p}$ value \\
\hline Cuff SBP (pre-assessment) [mm Hg] & $132 \pm 24$ & $133 \pm 21$ & $133 \pm 22$ & $131 \pm 25$ & 0.984 \\
\hline $\begin{array}{l}\text { Cuff SBP (waiting room — left arm) } \\
{[\mathrm{mm} \mathrm{Hg]}}\end{array}$ & $131 \pm 18$ & $130 \pm 16$ & $133 \pm 21$ & $132 \pm 24$ & 0.976 \\
\hline $\begin{array}{l}\text { Cuff SBP (waiting room — right arm) } \\
\text { [mm Hg] }\end{array}$ & $133 \pm 18$ & $132 \pm 17$ & $134 \pm 21$ & $131 \pm 26$ & 0.944 \\
\hline $\begin{array}{l}\text { Cuff SBP (waiting room — average) } \\
\text { [mm Hg] }\end{array}$ & $132 \pm 18$ & $131 \pm 16$ & $133 \pm 21$ & $132 \pm 24$ & 0.981 \\
\hline $\begin{array}{l}\text { Cuff SBP (pre-diagnostic cath) } \\
{[\mathrm{mm} \mathrm{Hg]}}\end{array}$ & $133 \pm 17$ & $131 \pm 17$ & $134 \pm 22$ & $133 \pm 26$ & 0.98 \\
\hline $\begin{array}{l}\text { Cuff SBP (simultaneous with intra- } \\
\text {-arterial brachial) [mm Hg] }\end{array}$ & $138 \pm 19$ & $138 \pm 27$ & $137 \pm 28$ & $136 \pm 31$ & 0.993 \\
\hline Cuff SBP (average of all) [mm Hg] & $133 \pm 18$ & $135 \pm 15$ & $134 \pm 22$ & $134 \pm 24$ & 0.996 \\
\hline Cuff PP (pre-assessment) [mm Hg] & $52 \pm 12$ & $51 \pm 13$ & $54 \pm 20$ & $51 \pm 12$ & 0.814 \\
\hline $\begin{array}{l}\text { Cuff PP (waiting room — left arm) } \\
{[\mathrm{mm} \mathrm{Hg]}}\end{array}$ & $52 \pm 12$ & $52 \pm 13$ & $55 \pm 14$ & $51 \pm 15$ & 0.651 \\
\hline $\begin{array}{l}\text { Cuff PP (waiting room — right arm) } \\
{[\mathrm{mm} \mathrm{Hg}]}\end{array}$ & $55 \pm 13$ & $54 \pm 13$ & $57 \pm 13$ & $52 \pm 13$ & 0.534 \\
\hline $\begin{array}{l}\text { Cuff PP (waiting room — average) } \\
{[\mathrm{mm} \mathrm{Hg}]}\end{array}$ & $53 \pm 12$ & $53 \pm 12$ & $57 \pm 14$ & $51 \pm 15$ & 0.472 \\
\hline $\begin{array}{l}\text { Cuff PP (pre-diagnostic cath) } \\
\text { [mm Hg] }\end{array}$ & $54 \pm 11$ & $52 \pm 13$ & $57 \pm 16$ & $51 \pm 13$ & 0.503 \\
\hline $\begin{array}{l}\text { Cuff PP (simultaneous with intra- } \\
\text {-arterial brachial) }[\mathrm{mm} \mathrm{Hg}]\end{array}$ & $57 \pm 13$ & $59 \pm 20$ & $57 \pm 18$ & $55 \pm 20$ & 0.889 \\
\hline Cuff PP (average of all) [mm Hg] & $54 \pm 10$ & $53 \pm 12$ & $56 \pm 13$ & $53 \pm 10$ & 0.805 \\
\hline
\end{tabular}

*One-way ANOVA was used. No post hoc analysis was done due to non-significant overall effect; PP — pulse pressure; SBP — systolic blood pressure

Since aortic BP is more closely related to cardiovascular risk relative to upper arm BP values [18], we suggest that patients in phenotypes III and IV might be subjected to a higher future risk due to underestimation of high BP. However, the lack of amplification of SBP and PP may contribute to this risk more than the isolated value at a certain arterial segment (e.g. aorta) as shown in some patients with end-stage renal disease [19]. On the other hand, it could be speculated that excessive SBP amplification itself may enhance end-organ damage at vulnerable sites but examining whether clinical outcomes would differ between various BP phenotypes should be the focus of a prospective cohort study with a large sample size enabling the power to detect associations.

Of course, the mechanisms underlying differences between the $4 \mathrm{BP}$ phenotypes were not addressed in the current study, but explanations do exist and are multifaceted. These differences may be related to the interplay between ventricular output (e.g. stroke volume, chronotropy and contractility), large conduit arterial wall characteristics (e.g. elas- ticity) and criteria of small resistance arteries [20]. For instance, ejection of a good cardiac output into a healthy vascular system i.e. central arteries with good compliance that buffers the rapid increase in SBP, could lead to a downstream SBP augmentation considering the progressive peripheral tapering of vessels coupled with decreased wall thickness but increased stiffness [21]. On the contrary, lower ventricular inotropy and increased stiffness of central arteries relative to peripheral ones could lead to a high aortic SBP to a level that surpasses any peripheral amplification effect. This is more common with older age. Interestingly, patients in phenotypes-III and IV in the current study (groups with little or no peripheral SBP amplification) were significantly older than those in phenotypes-I and II (groups with high level of peripheral SBP amplification).

Thus, considering this complex interplay underlying the phenomenon of SBP amplification, it becomes unsurprising for cuff BP devices to be far less than accurate for dissecting these subtle differences using a one-size-for-all cuff methods. However, we 
should stress that this study (among others) is not meant at all to undermine the clinical value of cuff BP measurement, but rather suggests that developments could be applied to this method in order to improve its accuracy so that it could be used one day as a risk refinement tool.

Some limitations to this study deserve mention, first, we did not include a dedicated intra-arterial pressure waveform analysis device and we did not use multiple cuff devices for BP measurements due to limited resources and lack of fund. Second, we used a fluid-filled system rather than solid catheters for intra-arterial BP measurement, and this may introduce few errors if the system was not carefully handled (for example, impingement of the arterial wall in small arteries). Finally, our findings are not generalizable outside the examined population (patients with a clinical indication for coronary angiography). Having said this, there is no compelling case that cuff BP devices would be necessarily inaccurate among all patients. For example, the observed 4 phenotypes may be much less distinct among younger healthy people with less advancing age and no disease affecting the vascular system such as diabetes. However, this is to be examined in further large-scale cohort studies.

\section{Conclusion}

This study confirms that distinct intra-arterial BP phenotypes do exist based on SBP central-to-peripheral amplification. A daily used validated cuff $\mathrm{BP}$ method failed to discriminate this. Improving quality of BP measurements in routine practice by taking this phenotypic variation into account should be a future research focus.

\section{Acknowledgment}

We express our deep gratitude for Dr. Mohamed Bendary, who inspired the statistical analysis of this work. We are also thankful to all technical staff who helped in intra-arterial BP waveform recording.

None.

\section{Funding}

\section{Conflict of interest}

The authors declare that they have no conflict of interest.

\section{Ethical approval}

All procedures performed in this study were in accordance with the ethical standards of the institutional research committee and with the 1964 Helsinki dec- laration and its later amendments or comparable ethical standards.

\section{Informed consent}

Informed consent was obtained from all individual participants included in the study.

\section{References}

1. Pickering TG, Hall JE, Appel LJ, et al. Council on High Blood Pressure Research Professional and Public Education Subcommittee, American Heart Association. Recommendations for blood pressure measurement in humans: an AHA scientific statement from the Council on High Blood Pressure Research Professional and Public Education Subcommittee. J Clin Hypertens (Greenwich). 2005; 7(2): 102-109, doi: 10.1111/j.1524-6175.2005.04377.x, indexed in Pubmed: 15722655.

2. Ettehad D, Emdin CA, Kiran A, et al. Blood pressure lowering for prevention of cardiovascular disease and death: a systematic review and meta-analysis. Lancet. 2016; 387(10022): 957-967, doi: 10.1016/ S0140-6736(15)01225-8, indexed in Pubmed: 26724178.

3. Bordley J, Connor CAR, Hamilton WF, et al. Recommendations for human blood pressure determinations by sphygmomanometers. Circulation. 1951; 4(4): 503-509, doi: 10.1161/01.cir.4.4.503, indexed in Pubmed: 14870262.

4. Picone DS, Schultz MG, Otahal P, et al. Accuracy of Cuff-Measured Blood Pressure: Systematic Reviews and Meta-Analyses. J Am Coll Cardiol. 2017; 70(5): 572-586, doi: 10.1016/j.jacc.2017.05.064, indexed in Pubmed: 28750701.

5. Benmira A, Perez-Martin A, Schuster I, et al. An ultrasound look at Korotkoff sounds: the role of pulse wave velocity and flow turbulence. Blood Press Monit. 2017; 22(2): 86-94, doi: $10.1097 /$ MBP.0000000000000231, indexed in Pubmed: 27902494.

6. Amoore JN. Oscillometric sphygmomanometers: a critical appraisal of current technology. Blood Press Monit. 2012; 17(2): 80-88, doi: 10.1097/MBP.0b013e32835026b0, indexed in Pubmed: 22228040

7. Picone DS, Schultz MG, Climie RED, et al. Aortic-to-brachial stiffness gradient and kidney function in type 2 diabetes. J Hypertens. 2016;34(6): 1132-1139, doi: 10.1097/HJH.0000000000000916, indexed in Pubmed: 27035734.

8. Redheuil A, Yu WC, Mousseaux E, et al. Age-related changes in aortic arch geometry: relationship with proximal aortic function and left ventricular mass and remodeling. J Am Coll Cardiol. 2011; 58(12): 1262-1270, doi: 10.1016/j.jacc.2011.06.012, indexed in Pubmed: 21903061.

9. Murgo JP, Westerhof N, Giolma JP, et al. Aortic input impedance in normal man: relationship to pressure wave forms. Circulation. 1980; 62(1): 105-116, doi: 10.1161/01.cir.62.1.105, indexed in Pubmed: 7379273.

10. Peng X, Schultz MG, Picone DS, et al. Arterial reservoir characteristics and central-to-peripheral blood pressure amplification in the human upper limb. J Hypertens. 2017; 35(9): 1825-1831, doi: $10.1097 /$ HJH.0000000000001400, indexed in Pubmed: 28505065.

11. Picone DS, Schultz MG, Peng X, et al. Discovery of New Blood Pressure Phenotypes and Relation to Accuracy of Cuff Devices Used in Daily Clinical Practice. Hypertension. 2018; 71(6): 1239-1247, doi: 10.1161/HYPERTENSIONAHA.117.10696, indexed in Pubmed: 29632105.

12. Sharman JE, Avolio AP, Baulmann J, et al. Validation of noninvasive central blood pressure devices: ARTERY Society task force consensus statement on protocol standardization. Eur Heart J. 2017; 38(37): 2805-2812, doi: https://dx.doi.org/10, indexed in Pubmed: 28158489.

13. El As, Topouchian JA, Darne BM, et al. Validation of the Omron HEM-907 device for blood pressure measurement. Am J Hypertension. 2002; 7(4): 237-241, doi: 10.1097/00126097-20020800000006, indexed in Pubmed: 12198340. 
14. Stergiou GS, Lourida P, Tzamouranis D. Replacing the mercury manometer with an oscillometric device in a hypertension clinic: implications for clinical decision making. J Hum Hypertens. 2011; 25(11): 692-698, doi: 10.1038/jhh.2010.107, indexed in Pubmed: 21107434.

15. O'Brien E, Atkins N, Stergiou G, et al. European Society of Hypertension International Protocol revision 2010 for the validation of blood pressure measuring devices in adults. Blood Press Monit. 2010; 15(1): 23-38, doi: 10.1097/mbp.0b013e3283360e98, indexed in Pubmed: 20110786.

16. Kannel WB, Gordon T, Schwartz MJ. Systolic versus diastolic blood pressure and risk of coronary heart disease. The Framingham study. Am J Cardiol. 1971; 27(4): 335-346, doi: 10.1016/00029149(71)90428-0, indexed in Pubmed: 5572576.

17. Prospective Studies Collaboration. Age-specific relevance of usual blood pressure to vascular mortality: a meta-analysis of individual data for one million adults in 61 prospective studies. Lancet. 2002; 360(9349): 1903-1913, doi: 10.1016/s0140-6736(02)11911-8, indexed in Pubmed: 12493255.
18. Vlachopoulos C, Aznaouridis K, Stefanadis C. Prediction of cardiovascular events and all-cause mortality with arterial stiffness: a systematic review and meta-analysis. J Am Coll Cardiol. 2010; 55(13): 1318-1327, doi: 10.1016/j.jacc.2009.10.061, indexed in Pubmed: 20338492.

19. Safar ME, Blacher J, Pannier B, et al. Central pulse pressure and mortality in end-stage renal disease. Hypertension. 2002; 39(3): 735-738, doi: $10.1161 /$ hy0202.098325, indexed in Pubmed: 11897754.

20. Avolio AP, Van Bortel LM, Boutouyrie P, et al. Role of pulse pressure amplification in arterial hypertension: experts' opinion and review of the data. Hypertension. 2009; 54(2): 375-383, doi: 10.1161/HYPERTENSIONAHA.109.134379, indexed in Pubmed: 19564542 .

21. Gaddum N, Alastruey J, Chowienczyk P, et al. Relative contributions from the ventricle and arterial tree to arterial pressure and its amplification: an experimental study. Am J Physiol Heart Circ Physiol. 2017; 313(3): H558-H567, doi: 10.1152/ajpheart.00844.2016, indexed in Pubmed: 28576835. 